\title{
Wireless near-infrared spectroscopy of skeletal muscle oxygenation and hemodynamics during exercise and ischemia
}

\author{
Babak Shadgan ${ }^{\mathrm{a}, *}$, W. Darlene Reid ${ }^{\mathrm{b}}$, Reza Gharakhanlou ${ }^{\mathrm{c}}$, Lynn Stothers ${ }^{\mathrm{d}}$ and \\ Andrew John Macnab ${ }^{\mathrm{d}}$ \\ ${ }^{a}$ Muscle Biophysics Laboratory, University of British Columbia, Vancouver, BC, Canada \\ ${ }^{\mathrm{b}}$ Muscle Biophysics Laboratory, Department of Physical Therapy, University of British Columbia, \\ Vancouver, BC, Canada \\ ${ }^{\mathrm{c}}$ Tarbiat Modares University, Tehran, Iran \\ ${ }^{\mathrm{d}}$ Faculty of Medicine, University of British Columbia, UBC Hospital Bladder Care Centre, Vancouver, \\ $B C$, Canada
}

\begin{abstract}
The majority of in vivo applications of near-infrared spectroscopic (NIRS) monitoring use continuous wave instruments that require a fiberoptic cable connection between the subject and the instrument during monitoring. In studies of muscle physiology where subjects are exercising, and particularly in those who are engaged in sports activity, a wireless instrument with telemetric capacity provides obvious advantages. Having access to reliable telemetric NIRS technology will also increase the practicality and scope of this biomedical monitoring technique in clinical settings.

We report the feasibility of using a wireless continuous wave NIRS instrument with light emitting diodes, spatially resolved configuration, and Bluetooth ${ }^{\circledR}$ capability to study skeletal muscle oxygenation and hemodynamics during isometric contraction and ischemia induction.

In ten healthy subjects comparable patterns of change in chromophore concentration (oxygenated and deoxygenated hemoglobin), total hemoglobin and muscle oxygen saturation were observed during 3 sets of isometric voluntary forearm muscle contraction at 10,30 and 50\% of maximum voluntary capacity (MVC), and a period of ischemia generated subsequently.

This small series indicates that data with good intra- and inter-subject reproducibility that is free of movement artifact can be obtained with the wireless NIRS instrument described. The validity of these muscle studies demonstrate a basis for applying wireless NIRS monitoring to other biomedical applications.
\end{abstract}

Keywords: NIRS, chromophore concentration, perfusion, oxygen consumption, isometric exercise, muscle ischemia, noninvasive measurement, telemetry

\section{Introduction}

Near-infrared spectroscopy (NIRS) is a well established optical technique that monitors changes in concentration of the chromophores oxyhemoglobin $\left(\mathrm{O}_{2} \mathrm{Hb}\right)$, deoxyhemoglobin $(\mathrm{HHb})$, total hemoglobin

\footnotetext{
${ }^{*}$ Corresponding author: Babak Shadgan, MD, MSc Sports Medicine, Muscle Biophysics Laboratory, University of British Columbia, Room 611-828, West 10th Avenue, Vancouver, BC, V5Z 1M9, Canada. Tel.: +1 604875 4111, ext. 66055; Fax: +1 604228 1158; E-mail: shadgan@gmail.com.
} 
$(\mathrm{tHb})$ and cytochrome (CCO) in a variety of tissues [1,2]. In the study of muscle, the science of NIRS utilizes the relative transparency of tissue to photons in the near-infrared (NIR) spectrum, and the oxygen-dependent absorption changes of these photons by hemoglobin $(\mathrm{Hb})$ and myoglobin $(\mathrm{Mb})$. Spectrum overlap prevents distinction between $\mathrm{Hb}$ and $\mathrm{Mb}$, but with continuous wave (CW) NIRS instruments using multiple NIR wavelengths, it is possible to use software algorithms to derive chromophore concentrations from raw optical data, and distinguish between oxy- and deoxy-hemoglobin/myoglobin $\left(\mathrm{O}_{2} \mathrm{Hb} / \mathrm{O}_{2} \mathrm{Mb}\right.$ and $\left.\mathrm{HHb} / \mathrm{HMb}\right)$. The feasibility of conventional CW NIRS in monitoring the pattern of skeletal muscle chromophore changes during rest, isometric exercise and ischemia is reported by different investigators [3-6]. NIRS has contributed new knowledge related to muscle physiology at a basic science level, is widely used as a measure of performance in exercise science, [7-9] and also to monitor muscle hypoxia and ischemia in sports medicine [10]. The non-invasive nature of the transcutaneous NIRS interface, and the ability to monitor continuously even during physical movement and active exercise provide an important means of measuring oxygenation and hemodynamics in muscle tissue in health and disease.

The majority of commercially available NIRS instruments are continuous wave spectrophotometers, and have proven reliability in the measurement of changes in $\mathrm{O}_{2} \mathrm{Hb}$ and $\mathrm{HHb}$. Multi-channel CW instruments can monitor more than one site simultaneously, and can be used for topographic mapping (fNIRS) when configured with a grid capable of holding multiple source-detectors and appropriate software. The fNIRS technique does not require strict motion restriction so is well suited for monitoring during normal activities including exercise [11]. CW instruments can use a variety of light sources including laser diodes with discrete wavelengths, a white light source combined with a CCD array and a grating to discriminate for wavelength [12], or light emitting diodes (LED). In spite of having a broader emission spectrum, LEDs have several advantages when compared to lasers, particularly their low cost and ability to be applied directly to the skin without the need for optical cables or lenses [13]. CW instruments detect light returning from tissue using photon counting hardware, usually consisting of a photodiode, photo multiplier tube or CCD. With CW technology the assumption is made that photon scatter in tissue is constant and a tissue specific differential path length factor (DPF) is used to calculate the optical path length from the inter optode distance [14].

The basic components of a CW NIRS instrument are: (a) a pulsed light source for each chromophore being sampled emitting light at a specific wavelength in the 729-920 nm NIR range; (b) fiberoptic bundles that transmit light from the source to a tissue interface (probe or patch) and back to the instrument's photon counting hardware; (c) an emitter and receiver in the tissue interface that introduce light into the tissue and receive the photons returning; (d) photon counting hardware; (e) computer with software containing algorithms for converting raw optical data into chromophore concentrations, storing and displaying data, and (f) a visual display where NIRS data are displayed numerically and/or graphically against time.

Many important studies of muscle have been completed using instruments configured in this way. However, refinements to this basic monitoring methodology have occurred and significant improvements and innovation continue to expand the research potential of NIRS. Some instruments provide the option to select wavelengths from multiple options; the ability to use more than one data channel to allow comparison between monitoring sites and/or tissue (e.g. opposite limbs, or to monitor muscle and brain simultaneously); or a signal weighted towards brain or muscle tissue (the former is achieved by subtracting a superficial signal from a deeper signal) $[15,16]$. Research instruments incorporate other technology such as phase modulation, or time resolved spectroscopy, and a range of instruments are 
now configured for spatially resolved spectroscopy (SRS). SRS incorporates multiple sensors at different distances from the emitter, which enables the ratio of oxygenated to total tissue hemoglobin to be measured and a quantitative measure of tissue oxygen saturation to be derived $[9,17,18]$. Innovations also now enable subjects actively exercising or participating in a range of sports to be successfully monitored [19].

CW instruments of small size, particularly those with telemetric capacity, represent an important advance in sports medicine and exercise physiology studies [1]. Instruments with wireless capability described include: a single-channel wearable NIRS system capable of monitoring brain activity in freely moving subjects [20,21]; a commercially available miniaturized device (Arquatis GmbH, Switzerland) that combines spatially resolved NIR spectroscopy with a 3D-accelerometer for muscle measurement during field training; a NIR imaging (NIRI) sensor incorporating 4 light sources and 4 detectors capable of a sampling rate of $100 \mathrm{~Hz}$ [13]; and a 3 wavelength single-channel CW reflectance instrument used to study muscle oxygenation in athletes [22].

The NIRI sensor was tested prior to brain study using a conventional arterial occlusion experiment to validate the functionality of the system. Changes in chromophore concentration in the brachioradialis muscle of a male volunteer comparable to data obtained with conventional instrumentation were obtained in response to 5 consecutive periods of ischemia. The feasibility of assessing hemodynamic changes in the cerebral cortex during thumb and index finger tapping was then demonstrated [13]. The 3 wavelength single-channel CW device was used successfully to study oxygenation in the quadriceps muscle of 10 athletes using 2 experimental protocols; a maximal output power experiment and three step incremental load exercise [22]. We report the use of a commercially available CW wireless NIRS instrument with paired LED light sources in a spatially resolved configuration to monitor forearm muscle oxygenation and hemodynamics in healthy subjects during exercise and tourniquet induced ischemia. The ease of use of this instrument and the reproducibility of the data obtained confirm the feasibility of using miniaturized, portable NIRS technology with telemetric capacity for human clinical studies.

\section{Methods}

\subsection{Instrumentation}

The instrument used was a compact, self-contained unit (PortaMon) developed by Artinis Medical Systems, BV, The Netherlands, that incorporates the following features. The unit measures $83 \times 52 \times$ $20 \mathrm{~mm}$, weighs $84 \mathrm{~g}$. It uses paired light emitting diodes with wavelengths of 760 and $850 \mathrm{~nm}$ as the NIR light source. Three pairs of these LEDs are mounted in a spatially resolved configuration so that the three light sources and one receiver provide 3 source-detector separation distances $(30,35$ and $40 \mathrm{~mm})$. The sensor is a silicone photodiode with ambient light protection. Data is collected at $10 \mathrm{~Hz}$. Power is supplied by a rechargeable Lithium Polymer battery with a capacity of about six hours of continuous monitoring. The unit has an internal memory with a capacity of $2 \mathrm{MB}$ to store data during ambulatory measurement, and incorporates Bluetooth ${ }^{\circledR}$ technology with broadcast range of $30 \mathrm{~m}$ to transfer data to a laptop computer for data analysis, graphic display and storage. The spatially resolved configuration of the emitters to the sensors provides pathlength geometry which makes it possible to also derive a measure of average tissue oxygen saturation using photon diffusion theory [23]. The instrument is attached to a subject by taping (Fig. 1).

Software controlling the device allows data interpretation at each source-detector distance, and derives changes in the concentration of the chromophores $\mathrm{O}_{2} \mathrm{Hb}$ and $\mathrm{HHb}$ from the raw optical data, total 


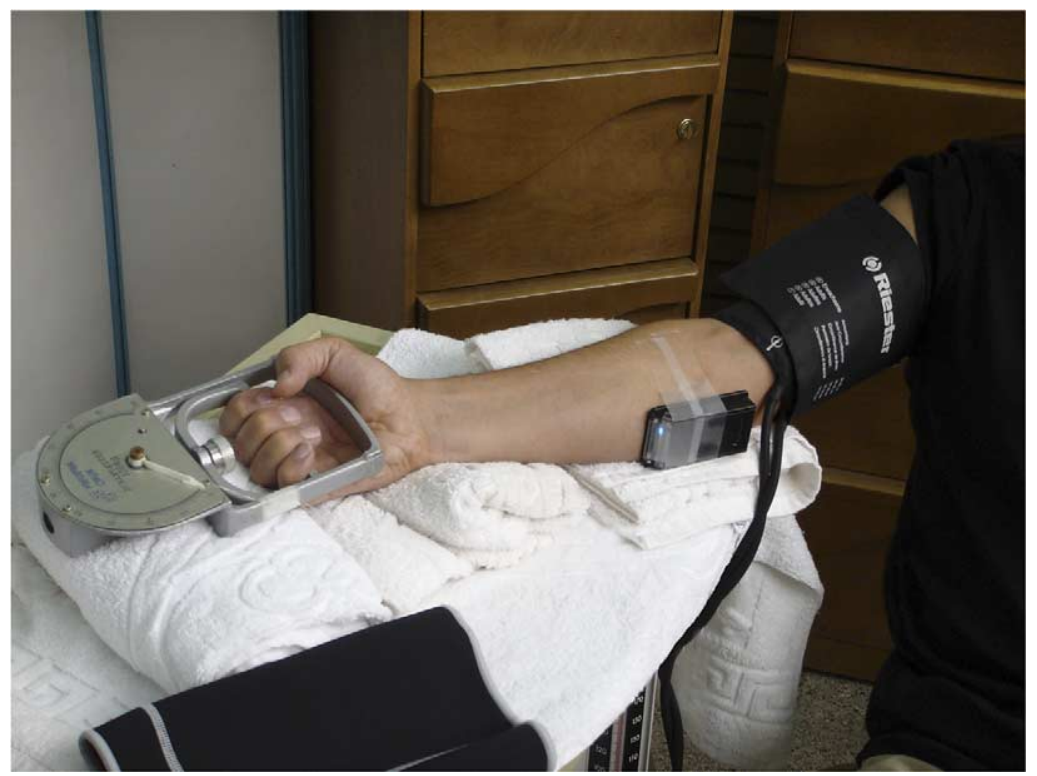

Fig. 1. The wireless instrument positioned over the flexor digitorum superficialis muscle for study of muscle oxygenation and hemodynamics.

hemoglobin $(\mathrm{tHb})$ as the sum of $\mathrm{O}_{2} \mathrm{Hb}$ and $\mathrm{HHb}$, and tissue oxygenation saturation index (TSI (\%)) in real time.

\subsection{Subjects}

Volunteer male athletes were recruited following interview to exclude any major co-morbidity and/or significant acute or chronic injury that could affect their ability to perform the forearm muscle contraction protocol required for the study. The study received ethical approval and all subjects provided informed consent.

\subsection{Protocol}

The equipment set up used on all subjects is shown in Fig. 1. Each subject sat in a chair with his right arm placed on a table with the elbow extended at the level of the heart, and the forearm positioned upward at 30 degrees with the hand resting on a handgrip dynamometer. Support was provided to maintain a stable position and an unrestricted circulation while leaving the forearm free. The mid-point of the belly of the flexor digitorum superficialis muscle was identified and marked. The skin-fold thickness at this location was measured using a skin-fold caliper, and the wireless NIRS device placed over the marked point and fixed by taping. A sphygmomanometer cuff was placed loosely around the upper arm in order for arterial occlusion to be applied as part of the experiment. Although the sensor in the instrument incorporates an ambient light filter, the forearm was covered by an opaque cloth to avoid any possibility of signal contamination by ambient light.

Maximum voluntary contraction force (MVC) was measured three times with one minute periods of rest in between, with the highest measurement considered the final MVC value. Values for 10, 30 and 
$50 \%$ of the final MVC were calculated and marked on the dynamometer display so as to be visible to the subject.

Following a 10 min rest period monitoring was begun with one minute of baseline and then continuous measurement throughout the remainder of the protocol at $10 \mathrm{~Hz}$. Each subject was asked to perform a series of $30 \mathrm{~s}$ sustained isometric handgrip contractions to the previously defined values of their MVC followed by 3 min periods of rest; first at $10 \%$ of MVC, then at 30\% MVC, and finally at $50 \%$ MVC. After $10 \mathrm{~min}$ of rest, the sphygmomanometer cuff was inflated to a pressure of $250 \mathrm{mmHg}$ and held at this pressure for $30 \mathrm{~s}$ to sustain forearm ischemia, and then deflated. NIRS monitoring was continued for a further $10 \mathrm{~min}$ and the experimental protocol then ended.

\section{Results}

Ten healthy male athletes participated. All subjects were right-handed. Table 1 shows their mean age, height and weight.

Similar patterns of change in $\mathrm{tHb}, \mathrm{O}_{2} \mathrm{Hb}, \mathrm{HHb}$ and TSI (\%) were seen in all ten subjects during the 3 sets of isometric voluntary forearm muscle contraction at 10,30 and 50\% of MVC, and the period of ischemia generated subsequently. Figure 2 is a composite graph of data from a typical subject which shows the pattern of change in chromophore concentration $\left(\mathrm{O}_{2} \mathrm{Hb}\right.$ and $\left.\mathrm{HHb}\right)$ and $\mathrm{tHb}$ observed over the course of the protocol, with TSI (\%) included below on the same time scale.

Each isometric contraction decreased muscle blood flow by increasing intramuscular pressure and compressing the small intramuscular blood vessels; $\mathrm{tHb}$ fell while $\mathrm{O}_{2} \mathrm{Hb}$ decreased and $\mathrm{HHb}$ increased. Some variation in the magnitude of change was demonstrated between subjects. Mean values $( \pm$ SD) for all 10 subjects of changes in $\mathrm{tHb}, \mathrm{O}_{2} \mathrm{Hb}, \mathrm{HHb}$ and TSI (\%) during 10, 30 and $50 \%$ of $\mathrm{MVC}$ and the subsequent period of ischemia are shown in Table 2.

During the ischemia phase of the protocol an initial increase in $\mathrm{tHb}$ was observed during the inflation of the pneumatic cuff, indicating blood flow congestion in the forearm. Once cuff pressure completely obstructed forearm blood flow, a sharp decrease in $\mathrm{O}_{2} \mathrm{Hb}$ and an equal and opposite increase in $\mathrm{HHb}$ were observed, while $\mathrm{tHb}$ tended to remain stable or slightly increase. An example of a typical tracing is shown in Fig. 3, which also shows a pattern indicative of microvascular arterial pulsation before tourniquet inflation and immediately after tourniquet deflation.

The changes observed in the value of muscle TSI (\%) differed in response to 10,30 and $50 \%$ of MVC and during induced ischemia. Tissue muscle oxygen saturation declined the greatest amount during 30 and 50\% of MVC. This was a consistent finding in all subjects. Variation in the values obtained was somewhat greater during isometric contraction at $10 \%$ MVC than during ischemia (Table 2). This finding is in accordance with the previous experiments with conventional CW NIRS instruments which demonstrated that sustained exercise above 30\% MVC induces a complete ischemia [24,25].

Table 1

Mean $( \pm \mathrm{SD})$ physical characteristics of subjects

\begin{tabular}{lcc}
\hline Subjects & Mean & SD \\
\hline Age & 30.7 & 6.2 \\
Weight $(\mathrm{kg})$ & 79.1 & 6.7 \\
Height $(\mathrm{cm})$ & 177 & 2.7 \\
Skinfold thickness $(\mathrm{mm})$ & 6.8 & 1.8 \\
\hline
\end{tabular}



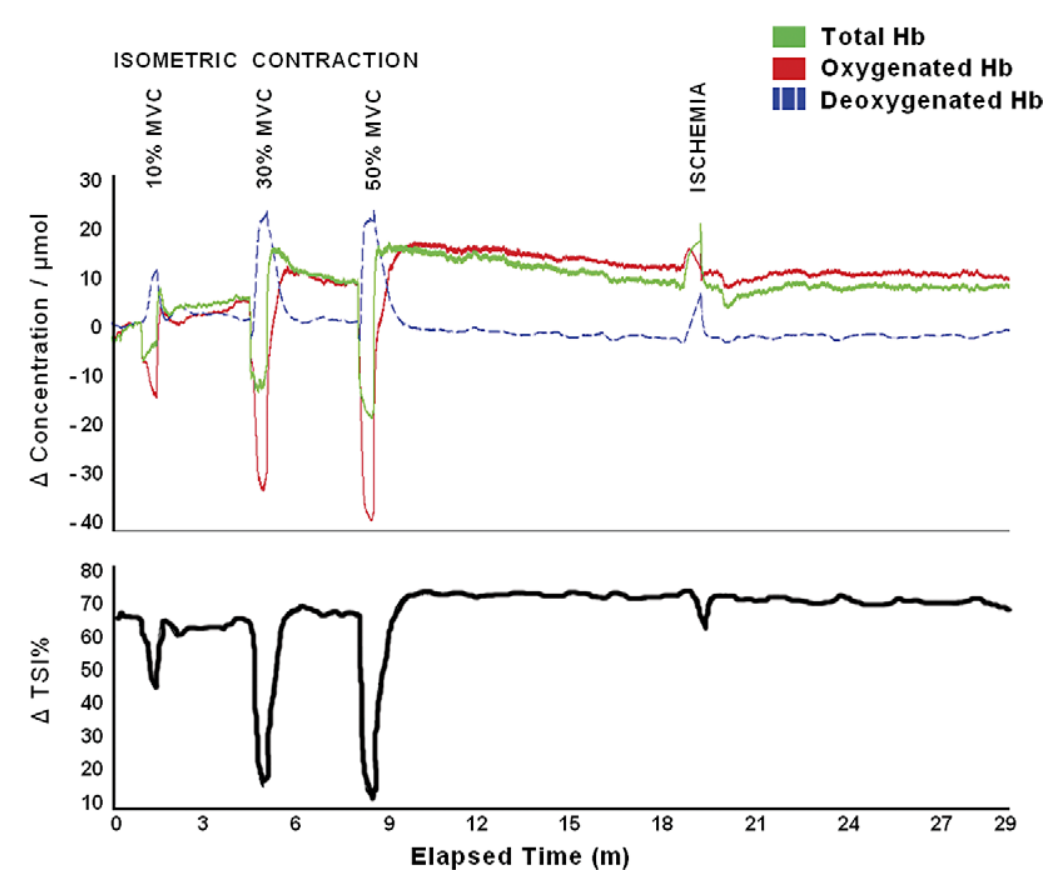

Fig. 2. An example of the pattern of change in chromophore concentration $\left(\mathrm{O}_{2} \mathrm{Hb}\right.$ and $\left.\mathrm{HHb}\right)$ and $\mathrm{tHb}$ and TSI $(\%)$ over the experimental protocol.

Table 2

Mean $( \pm \mathrm{SD})$ changes of $\mathrm{O}_{2} \mathrm{Hb}, \mathrm{HHb}$ and $\mathrm{tHb}$ along with TSI (\%) during 10,30 and $50 \%$ of MVC and ischemia

\begin{tabular}{lcc}
\hline & Mean/ $\mu \mathrm{mol}$ & $\mathrm{SD}$ \\
\hline$\Delta \mathrm{O}_{2} \mathrm{Hb}-10 \% \mathrm{MVC}$ & -8 & 5.3 \\
$\Delta \mathrm{O}_{2} \mathrm{Hb}-30 \% \mathrm{MVC}$ & -25.7 & 13.5 \\
$\Delta \mathrm{O}_{2} \mathrm{Hb}-50 \% \mathrm{MVC}$ & -35.9 & 11.6 \\
$\Delta \mathrm{O}_{2} \mathrm{Hb}-\mathrm{Ischemia}$ & -5.7 & 3.7 \\
$\Delta \mathrm{HHb}-10 \% \mathrm{MVC}$ & 6.4 & 4.8 \\
$\Delta \mathrm{HHb}-30 \% \mathrm{MVC}$ & 15.5 & 5.6 \\
$\Delta \mathrm{HHb}-50 \% \mathrm{MVC}$ & 16.7 & 5 \\
$\Delta \mathrm{HHb}-\mathrm{Ischemia}$ & 5 & 1.9 \\
$\Delta \mathrm{tHb}-10 \% \mathrm{MVC}$ & -1 & 4.3 \\
$\Delta \mathrm{tHb}-30 \% \mathrm{MVC}$ & -9.7 & 9.5 \\
$\Delta \mathrm{tHb}-50 \% \mathrm{MVC}$ & -19.2 & 7.2 \\
$\Delta \mathrm{tHb}-\mathrm{Ischemia}$ & -0.3 & 3.6 \\
$\Delta \mathrm{TSI}(\%)-10 \% \mathrm{MVC}$ & -12.2 & 6.6 \\
$\Delta \mathrm{TSI}(\%)-30 \% \mathrm{MVC}$ & -37 & 7.9 \\
$\Delta \mathrm{TSI}(\%)-50 \% \mathrm{MVC}$ & -50.3 & 2 \\
$\Delta \mathrm{TSI}(\%)-$ Ischemia & -8.4 &
\end{tabular}

The technical features and software provided with the instrument made it straightforward to use. Reliable attachment to the subject was readily achieved and data was transferred to the laptop without any problems. Clear signals, free of noise or movement artifact were obtained in all subjects studied. Sub- 


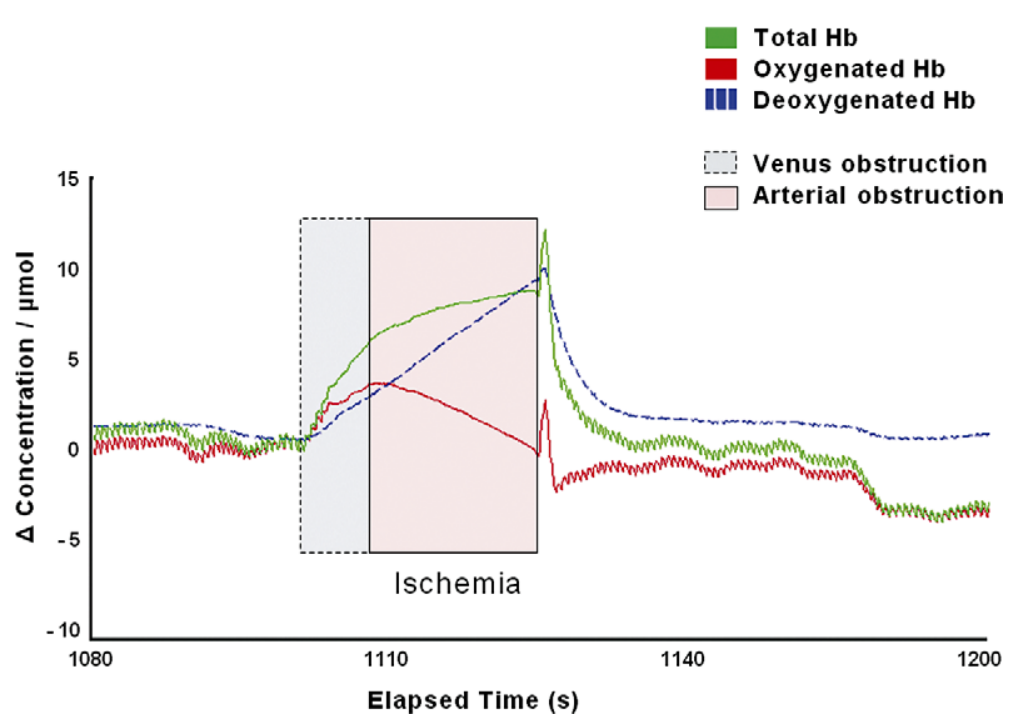

Fig. 3. An example of a typical pattern of tourniquet induced muscle ischemia which indicates microvascular arterial pulsation before tourniquet inflation and immediately after tourniquet deflation.

jects found the device comfortable to wear during the period of study; and the compact nature of the instrument and freedom from optical cables facilitated the set up and conduct of each element of the study protocol.

\section{Discussion}

We have demonstrated the feasibility of monitoring changes in chromophore concentration in the forearm muscle of healthy male subjects during sequential isometric contractions and a period of induced ischemia using a wireless NIRS instrument. Excellent, comparable patterns and magnitude of change were seen in the NIRS chromophore concentration data across subjects.

A significant decrease in $\mathrm{tHb}$ and $\mathrm{O}_{2} \mathrm{Hb}$ accompanied by an increase in $\mathrm{HHb}$ occurred in each subject during isometric contraction, and the magnitude of change became larger as the percentage of maximum voluntary contraction increased. During the period of induced forearm ischemia all subjects showed a similar pattern of increase in $\mathrm{HHb}$ and decrease in $\mathrm{O}_{2} \mathrm{Hb}$. The pattern and magnitude of change in muscle oxygen saturation (TSI (\%)) in response to incremental isometric contraction and subsequent arterial obstruction were also consistent and very comparable in all subjects. Our results also match those observed in prior studies involving muscle using conventional CW NIRS instruments by Kahn [24] and van Beekvelt [25]. Even the subtle changes of the initial rise in tHb and tendency for a gradual increase to continue during the first minute following cuff inflation have been observed in other studies [25].

The effects of ischemia on the patterns of change in $\mathrm{O}_{2} \mathrm{Hb}$ and $\mathrm{HHb}$ in our subjects are also consistent with those observed in other tissue such as the brain, spinal cord and bladder [17,26,27]. The changes in muscle TSI (\%) observed differed in response to 10, 30 and 50\% of MVC and during induced ischemia, declining most during 30 and 50\% of MVC. This finding is in accordance with the previous NIRS experiments which demonstrated that sustained exercise above 30\% MVC induces complete ischemia [24,25]. Each of these observations supports the validity of the NIRS data we obtained, which in turn 
confirms the precision of the LED light source and integrity of the technical design of the wireless instrument, and the accuracy of the algorithm it incorporates.

We recognize limitations in our study including our data being limited to individual measurements in ten subjects. However, the wireless instrument monitored reliably on each occasion; was sensitive enough to detect normal arterial pulsation; was comfortable and unobtrusive to wear; was simple to use; and generated highly reproducible data in all subjects. Our experience and results from this feasibility study advance the potential for wireless NIRS technology to contribute to studies of basic muscle physiology and performance assessment in sports medicine. This is the first report of a prospective series of NIRS measurements of muscle oxygen saturation using a wireless device with dual wavelengths in multiple subjects, and adds to the prior report of a single forearm measurement [13] and to the observations by Liu and colleagues with a single channel prototype instrument in 10 athletes [22].

We have described our successful use of this commercially available wireless NIRS instrument in the belief that other investigators will be able to design experiments that benefit from this technology. Wireless monitoring is a significant advance that will contribute to multiple future applications of NIRS in a variety of tissues where changes in oxygenation and hemodynamics are clinically significant. Independent trials are required to validate our findings. However, we believe that investigators conducting such trials will rapidly expand the use of this device and the technology it incorporates to other studies, particularly when their research questions relate to the study of skeletal muscle, sports medicine and exercise physiology.

\section{Acknowledgements}

The authors wish to thank National Olympic \& Paralympic Academy of Iran for their collaboration in this study. B. Shadgan was supported in part by a Senior Graduate Trainee Award from the Michael Smith Foundation for Health Research (MSFHR).

\section{References}

[1] M. Wolf, M. Ferrari and V. Quaresima, Progress of near-infrared spectroscopy and topography for brain and muscle clinical applications, J. Biomed. Opt. 12(6) (2007), 062104.

[2] T. Hamaoka, K.K. McCully, V. Quaresima, K. Yamamoto and B. Chance, Near-infrared spectroscopy/imaging for monitoring muscle oxygenation and oxidative metabolism in healthy and diseased humans, J. Biomed. Opt. 12(6) (2007), $0621051-12$.

[3] M.C.P. van Beekvelt, B.G.M. van Engelen, R.A. Wevers and W.J.M. Colier, In vivo quantitative near-infrared spectroscopy in skeletal muscle during incremental isometric handgrip exercise, Clin. Physiol. Funct. Imaging 22 (2002), 1-8.

[4] R.A. De Blasi, M. Cope, C. Elwell, F. Safoue and M. Ferrari, Noninvasive measurement of human forearm oxygen consumption by near infrared spectroscopy, Eur. J. Appl. Physiol. Occup. 67 (1993), 20-25.

[5] A. Usaj, B. Jereb, P. Robi and S.P. von Duvillard, The influence of strength-endurance training on the oxygenation of isometrically contracted forearm muscles, Eur. J. Appl. Physiol. 100(6) (2007), 685-692.

[6] R. Kime, T. Hamaoka, T. Sako, M. Murakami, T. Homma, T. Katsumura and B. Chance, Delayed reoxygenation after maximal isometric handgrip exercise in high oxidative capacity muscle, Eur. J. Appl. Physiol. 89(1) (2003), 34-41.

[7] V. Quaresima, R. Lepanto and M. Ferrari, The use of near infrared spectroscopy in sports medicine, J. Sport Med. Phys. Fitness 43(1) (2003), 1-13.

[8] M.I.R. Pereira, P.S.C. Gomes and Y.N. Bhambhani, A brief review of the use of near infrared spectroscopy with particular interest in resistance exercise, Sports Med. 37(7) (2007), 615-624.

[9] R. Boushel, H. Langberg, J. Olesen, J. Gonzales-Alonzo, J. Bulow and M. Kjaer, Monitoring tissue oxygen availability with near infrared spectroscopy (NIRS) in health and disease, Scand. J. Med. Sci. Sports 11 (2001), 213-222.

[10] J.G. van den Brand, E.J. Verleisdonk and C. van der Werken, Near infrared spectroscopy in the diagnosis of chronic exertional compartment syndrome, Am. J. Sport Med. 32(2) (2004), 452-456. 
[11] Y. Hoshi, Functional near-infrared spectroscopy: current status and future prospects, J. Biomed. Opt. 12(6) (2007), 062106.

[12] M. Cope, D.T. Delpy, S. Wray, J.S. Wyatt and E.O.R. Reynolds, A CCD spectrometer to quantitate the concentration of chromophores in living tissue utilizing the absorption peak of water at $975 \mathrm{~nm}$, Adv. Exp. Med. Biol. 248 (1989), 33-40.

[13] T. Muehlemann, D. Haensse and M. Wolf, Wireless miniaturized in vivo near infrared imaging, Opt. Expr. 16(14) (2008), 10323-10330.

[14] D.T. Delpy, M. Cope, P. van der Zee, S. Arridge, S. Wray and J.S. Wyatt, Estimation of optical path length through tissue from direct time of flight measurements, Phys. Med. Biol. 33 (1988), 1433-1442.

[15] P.W. McCormick, M. Stuart, M.G. Goetting and G. Balakrishnan, Regional cerebrovascular oxygen saturation measured by optical spectroscopy in humans, Stroke 22 (1991), 596-602.

[16] M.C. van der Sluijs, N.J.M. Colier, R.J.F. Houston and B. Oesburg, A new and highly sensitive continuous wave near infrared spectrophotometer with multiple detectors, Proc. Soc. Photo Opt. Instrum. Eng. 3194 (1997), 63-72.

[17] M. Ferrari, L. Mottola and V. Quaresima, Principles, techniques and limitations of near infrared spectroscopy, Can. J. Appl. Physiol. 29 (2004), 463-487.

[18] P. Rolfe, In vivo near-infrared spectroscopy, Annu. Rev. Biomed. Eng. 2 (2000), 715-754.

[19] J.P. Neary, Application of near infrared spectroscopy to exercise sports science, Can. J. Appl. Physiol. 29(4) (2004), 488-503.

[20] Y. Hoshi and S.J. Chen, New dimensions of cognitive neuroscience research with near-infrared spectroscopy: Free motion neuroimaging studies, in: Progress in Brain Mapping Research, F.J. Chen, ed., Nova Science, New York, 2006, pp. 205229.

[21] T. Shiga, K. Yamamoto, K. Tanabe, Y. Nakase and B. Chance, Study of an algorithm based on model experiments and diffusion theory for a portable tissue oximeters, J. Biomed. Opt. 2 (1997), 154-161.

[22] F. Liu, Q. Luo, G. Xu and P. Li, Noninvasive detection of changes in skeletal muscle oxygenation during incremental exercise with near-infrared spectroscopy, Proc. Soc. Photo Opt. Instrum. Eng. 5254 (2003), 573-579.

[23] S. Suzuki, S. Takasaki, T. Ozaki and Y. Kobayashi, A tissue oxygenation monitor using NIR spatially resolved spectroscopy, Proc. Soc. Photo Opt. Instrum. Eng. 3597 (1999), 582-592.

[24] J.F. Kahn, J.C. Jouanin, J.L. Bussiere, E. Tinet, S. Avrillier, J.P. Ollivier and H. Monod, The isometric force that induces maximal surface muscle deoxygenation, Eur. J. Appl. Physiol. 78 (1998), 183-187.

[25] M.C.P. van Beekvelt, K. Orbon, B.G.M. van Engelen, R.A. Wevers and W.N.J.M. Colier, NIR spectroscopic measurement of local muscle metabolism during rhythmic, sustained and intermittent handgrip exercise, Proc. Soc. Photo Opt. Instrum. Eng. 5138 (2003), 35-45.

[26] A.J. Macnab, R.E. Gagnon, F. Gagnon and J.G. Leblanc, NIRS monitoring of brain and spinal cord - detection of adverse intra-operative events, Spectroscopy 17 (2003), 483-490.

[27] L. Stothers, B. Shadgan and A.J. Macnab, Urological applications of near infrared spectroscopy, Can. J. Urol. 15(6) (2008), 4399-4409. 


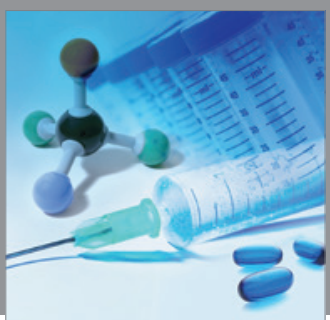

International Journal of

Medicinal Chemistry

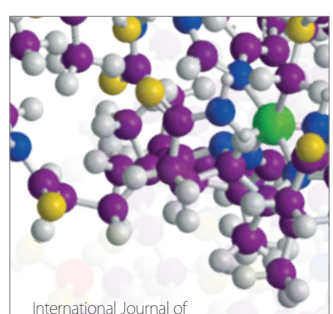

Carbohydrate Chemistry

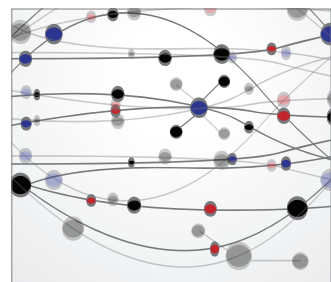

The Scientific World Journal
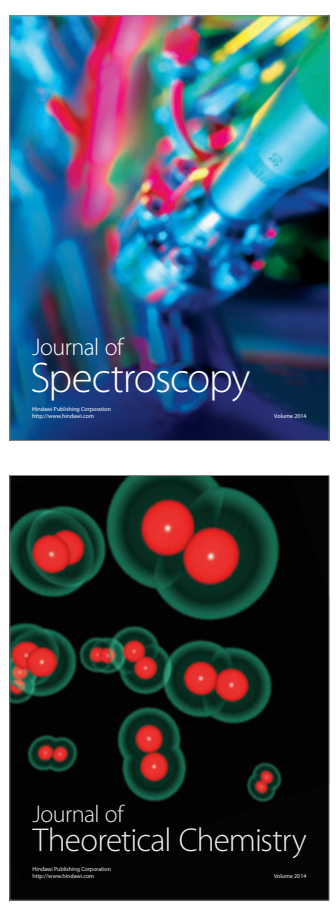
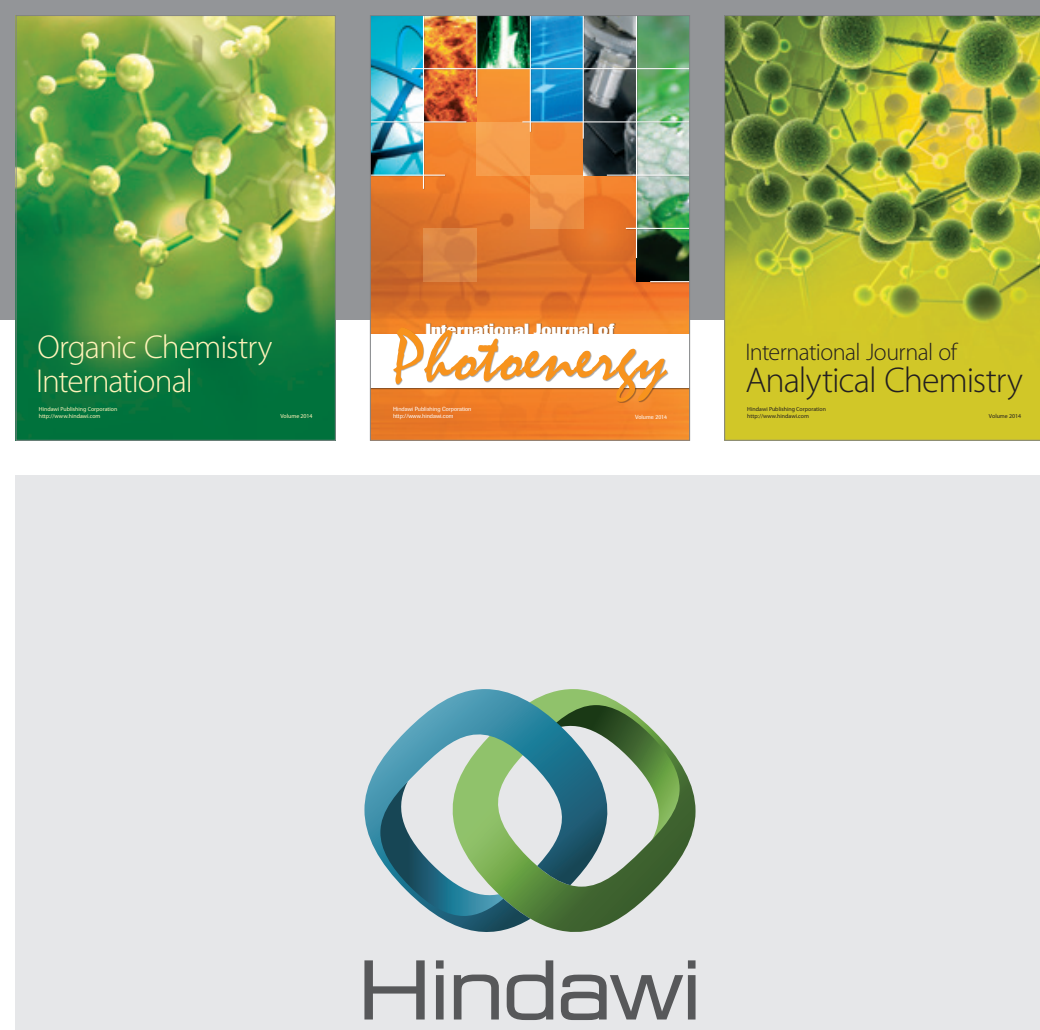

Submit your manuscripts at

http://www.hindawi.com
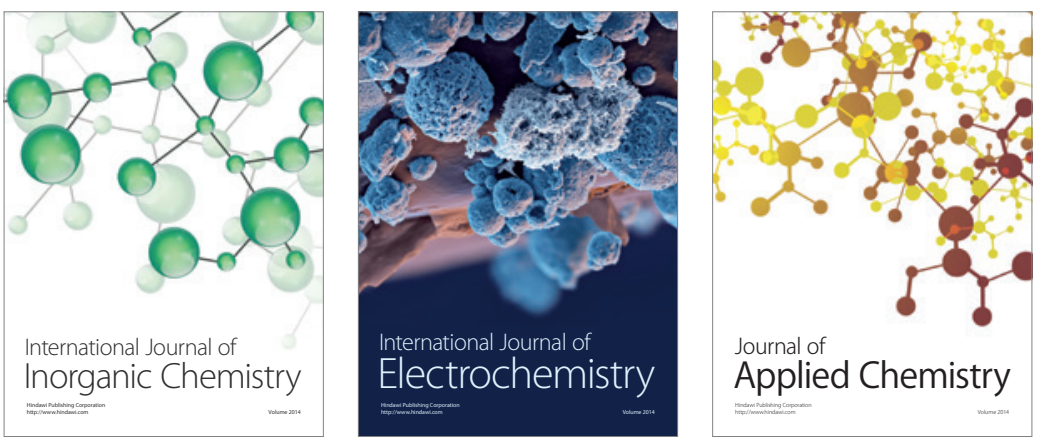

Journal of

Applied Chemistry
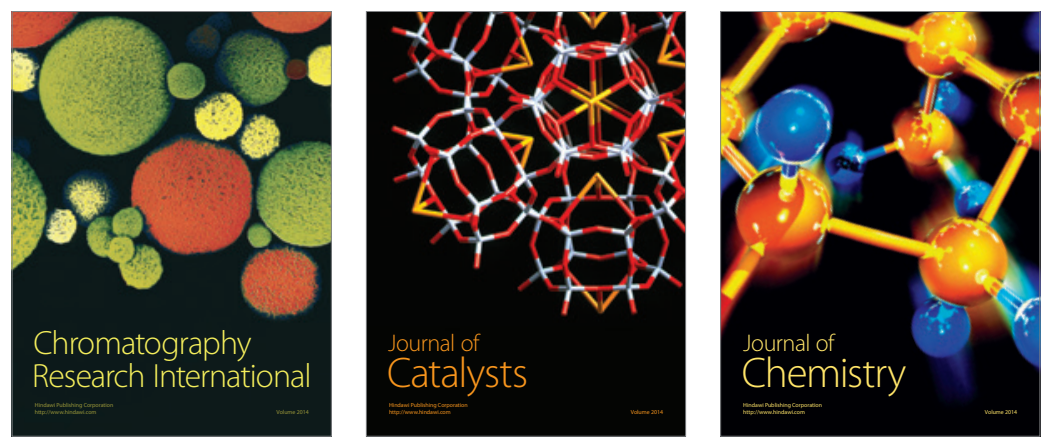
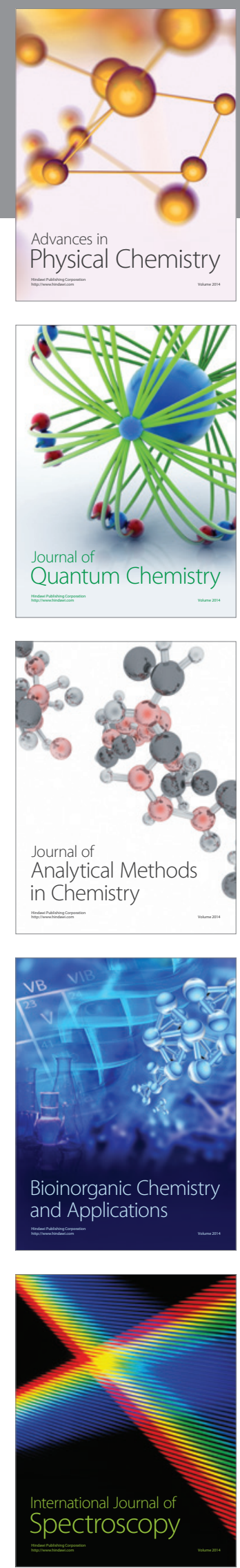